\title{
The global structure of computably enumerable sets
}

\author{
Peter A. Cholak
}

\begin{abstract}
We will work in the structure of the computably enumerable sets. The language is just inclusion, $\subseteq$. This structure is called $\mathcal{E}$. Our quest is to partially survey our current understanding of the global structure of $\mathscr{E}$ and the relationship between $\mathcal{E}$ and the computably enumerable degrees and to pose questions whose answers should provide further insight.
\end{abstract}

\section{Introduction}

There are four themes that run throughout most work on $\mathcal{E}$ : Definability, orbits/automorphisms/isomorphic substructures, computational complexity, and dynamic properties. Clearly, definability is a general theme throughout all of mathematical logic. The study of orbits and automorphisms (in general) dates from the 1870's and earlier. Mainly we will use Turing reducibility, Turing jump and the related jump classes (low and high ${ }_{n}$ classes) as a measure of computational complexity. This is part of our connection to the computably enumerable degrees. The dynamic properties are more hidden but we will try to highlight them. What is so nice in $\mathscr{E}$ is how all four themes interrelate with each other. At present this is just a bold claim but, in this paper, we will provide elaboration and justification of this claim.

1.1. Preliminaries. Before we start this journey we must deal with some preliminaries. First, this is not the only paper on $\mathcal{E}$ in this collection. Soare [2000] also focuses on $\mathcal{E}$.

All sets will be computably enumerable noncomputable sets and all degrees will be computably enumerable and noncomputable, unless otherwise noted. Our notation and definitions are standard and follow Soare [1987]; however we will try to provide some definitions and notational niceties throughout the text so the reader need not consult Soare [1987].

The sets and relations $0,1, \cap$ and $\cup$ are definable from $\subseteq$. Hence $\mathcal{E}$ can be considered as a lattice. " $X$ is computable" is definable; " $X$ is computable" iff $X$ is complemented. " $X$ is finite" is definable in $\mathcal{E}$; " $X$ is finite" iff all subsets of $X$ are computable (it takes a little computability theory to show if $X$ is infinite then $X$ has an infinite noncomputable subset).

1991 Mathematics Subject Classification. Primary 03D25.

Key words and phrases. Computably enumerable sets, open questions.

Research partially supported by NSF Grant DMS-96-34565.

(C)0000 (copyright holder) 
We will also consider the quotient structure $\mathscr{E}$ modulo the ideal of finite sets, $\mathscr{E}^{*} . \mathscr{E}^{*}$ is a definable quotient structure of $\mathscr{E}$. We use $A^{*}$ to denote the equivalence class of $A$ modulo the ideal of finite sets. If there is an automorphism, $\Phi^{*}$, of $\mathcal{E}^{*}$ such that $\Phi^{*}\left(A^{*}\right)=B^{*}$, then there is an automorphism, $\Phi$, of $\varepsilon$ such that $\Phi(A)=B$ (in which case we say $A$ and $B$ are automorphic) [see Soare, 1987, XV.2.7]. Given this, we will blur the difference between $\mathcal{E}$ and $\mathcal{E}^{*}$.

\section{A beautiful example}

Perhaps the example we are about to present is overworked but it still highlights our four themes in an excellent fashion.

$M$ is maximal if for all $W$ either $W \subseteq^{*} M$ or $M \cup W={ }^{*} \omega$. Friedberg [1958] showed that there is a maximal set [see Soare, 1987, X.3.3].

THeorem 2.1 (Soare [1974]). If $M_{1}$ and $M_{2}$ are maximal sets then there is an automorphism $\Phi$ of $\mathcal{E}$ such that $\Phi\left(M_{1}\right)=M_{2}$. Hence the maximal sets form an orbit.

Theorem 2.2 (Martin [1966]). A degree $\mathbf{h}$ is high iff there is a maximal set $M$ such that $M \in \mathbf{h}$.

Definition 2.3. A class $\mathscr{D}$ of degrees is invariant if there is a class $\&$ of (c.e.) sets such that

(i) $\mathbf{d} \in \mathscr{D}$ implies there is a $W$ in $\&$ and $\mathbf{d}$,

(ii) $W \in \&$ implies $\operatorname{deg}(W) \in \mathscr{D}$, and

(iii) $\&$ is closed under automorphic images.

COROLlary 2.4. The high degrees are invariant.

Let's see how our four themes come into play. Theorem 2.1 implies that the maximal sets are a nonempty elementarily definable orbit. (By elementarily definable we will mean definable by a formula in $\mathcal{L}_{\omega, \omega}$, an elementary formula.) Clearly, Corollary 2.4 involves computational complexity (measured in terms of jump classes). The proofs of Theorems 2.1 and 2.2 rely on dynamic properties.

We say that a function $f$ is dominant if $f$ dominates every computable function. The proof of Theorem 2.2 breaks up into two parts: First one shows that the principal function of the complement of a maximal set is a dominant function, and then, one shows that a computably enumerable degree $\mathbf{d}$ is high iff $\mathbf{d}$ contains a dominant function. The idea is that lots of numbers must enter a maximal set.

The proof of Theorem 2.1 relies, in part, on the following: There is a list of computably enumerable sets $U_{i}$ where $U_{i}=W_{f(e)}$ and $f$ is computable in $\mathbf{0}^{\prime \prime}$ such that

- for all $e$ there is an $i$ such that $W_{e}={ }^{*} U_{i}$,

- for all $e$ there is a $\sigma_{e}$ such that $\sigma_{e}$ is the $e$-state of $\bar{M}$ w.r.t. $\left\{U_{i}\right\}_{i \in \omega}(\sigma(i)=1$ iff $\bar{M} \subseteq{ }^{*} U_{i}$ ) [dymanic isomorphism], and

- if $X_{\sigma}=\cap\left\{U_{i}: \sigma(i)=1\right\}$ then $X_{\sigma} \searrow M=\left\{x: \exists s, t\left[x \in\left(X_{\sigma, s}-M_{s}\right) \cap M_{t}\right]\right\}$ is infinite iff $\sigma=\sigma_{e}$, for some $e$ (or an empty extension of some $\sigma_{e}$ ) [replete].

These facts can be established using the fact that $M$ is maximal. Several proofs of this can be found, all using some version of Soare's Extension Theorem [see Soare, 1987, XV.4.5]. For example, see Soare [1987, XV.4-6] or Cholak [1994b]. Soare has announced another proof of this using another version of the Extension Theorem which he is calling the New Extension Theorem, see Soare [2000] for more details. 


\section{Orbits and 1-types}

The creative sets (Harrington, see Soare [1987, XV.1.4]); the maximal sets; the quasimaximal sets of rank $k$, for any $k$ (Soare, see Soare [1987, XV.4.8]); the Herrmann sets (Herrmann, see Cholak and Downey [n.d.]); the quasi-Herrmann sets of rank $k$, for any $k$ (Cholak and Downey [n.d.]); the hemi-maximal sets (Downey and Stob [1990]); the hemiquasi-maximal sets of rank $k$, for any $k$ (Downey and Stob [1990]); the hemi-Herrmann sets (Cholak and Downey [n.d.]); and the hemi-quasi-Herrmann sets of rank $k$, for any $k$, (Cholak and Downey [n.d.]) are all elementarily definable orbits. (The actual definition of these sets is not important, just the fact that they are elementarily definable.) Since each of these orbits is elementarily definable, the defining formula describes a principal type. Hence there are infinitely many 1 -types of $\mathcal{E}^{*}$ and so $\mathcal{E}^{*}$ is not $\aleph_{0}$-categorical. Clearly $\mathcal{E}$ has infinitely many 1 -types: "X has $n$ elements" is a 1-type in $\mathcal{E}$.

QUESTION 3.1. (i) Show that these (the above list) are the only elementarily definable orbits.

(ii) Show these are the only principal types.

(iii) Describe all orbits.

The key to all of the above results is that we have some control over the complement of the set. So to show that the above list is not the complete list of elementarily definable orbits, one should examine the sets where one has some control over the complement.

A set $H$ is hhsimple iff $\mathcal{L}^{*}(H)$ (the supersets of $H$ modulo the finite sets) is a Boolean algebra. In general, the hhsimple sets will not work to extend the above list. As there are nonautomorphic hhsimple sets whose $\mathcal{L}^{*} \mathrm{~s}$ are isomorphic. This is due to Lerman, Shore and Soare [1978] and was later extended to all possible $\mathscr{L}^{*}$ s (for all hhsimple sets) by Herrmann [1989]. So given hhsimple sets whose $\mathcal{L}^{*} \mathrm{~s}$ are isomorphic, one could try to add some additional properties to ensure that the sets are automorphic. Some other good possibilities to extend the above list are $r$-maximal sets with a maximal superset and the major subsets of a maximal set.

However, I conjecture the Question 3.1 has a positive answer; there are no more such orbits. Even if this conjecture is incorrect, I doubt there is a first-order elementarily definable orbit whose proof is not similar to any of the known results. The next theorem shows that answering Question 3.1 is bound to be hard and supports my conjecture that the answer to Question 3.1 is positive.

Theorem 3.2 (Cholak, Downey and Harrington [n.d.]). $\{(A, B): A \simeq B\}$ is $\Sigma_{1}^{1}$ complete.

COROllary 3.3. (i) Not all orbits are elementarily definable.

(ii) $\mathbb{E}$ is not a prime model (of its theory).

(iii) There is no arithmetical description of all orbits.

(iv) The Scott rank of $\mathcal{E}$ is at least $\omega_{1}^{C K}$.

But can we actually find examples realizing and extending the above corollary? I.e.:

QUESTION 3.4. (i) Find an orbit which is not a principal orbit.

(ii) For all computable ordinal $\alpha$, find a pair of computably enumerable sets which are $\Delta_{\alpha}^{0}$-automorphic but not $\Delta_{\beta}^{0}$-automorphic, for $\beta<\alpha$. (The definition of $\Delta_{\alpha}^{0}$-automorphic appears below.)

(iii) Can we find an orbit $O$ such that membership in $O$ is $\Sigma_{1}^{1}$ complete? 
Examining types allows us to prove two model-theoretic results: $\mathbb{E}$ is not $\aleph_{0}$-categorical and is not a prime model. Since, if a model is homogeneous, its Scott rank is at most $\omega+1, \varepsilon^{*}$ is not homogeneous or saturated.

\section{Automorphisms}

The main question here is :

QueSTION 4.1. Describe all automorphisms of $\mathcal{E}$.

However, given the following theorem and Theorem 3.2, this seems like an unanswerable question. But we will argue that it is actually an answerable question.

Theorem 4.2 (Lachlan, see Soare [1987, XV.2.2]). There are $2^{\aleph_{0}}$ automorphisms of $\mathcal{E}$

We can sidestep Lachlan's result since all these automorphisms are similar. They just move computably enumerable subsets of almost cohesive sets. There is a tower of computable sets $R_{i}$ such that for every $e$, either $W_{e} \subseteq R_{e}$ or $W_{e} \subseteq \bar{R}_{e}$. Let $S_{n}$ be an infinite coinfinite computably enumerable subset of $R_{n+1}-R_{n}$. Given a function $f$, let $\Phi_{f}$ be the automorphism of $\mathcal{E}$ induced by sending $\Phi_{f}\left(S_{n}\right)$ to $\left(R_{n+1}-R_{n}\right)-S_{n}$ and $\Phi_{f}\left(\left(R_{n+1}-R_{n}\right)-S_{n}\right)$ to $S_{n}$ if $f(n)=1$; otherwise $\Phi_{f}$ (actually the permutation which induces $\left.\Phi_{f}\right)$ is the identity on $R_{n+1}-R_{n}$. But even without Lachlan's result we still would not have an arithmetical description of all automorphisms by Theorem 3.2.

Our understanding of automorphisms of $\mathcal{E}$ is unique to $\mathcal{E}$. In most structures with nontrivial automorphisms we can construct automorphisms via the normal "back and forth" argument. But this is not the case with $\mathcal{E}$. To construct automorphisms of $\mathcal{E}$ we use the properties of being well-visited and well-resided. Well-visited is $\Pi_{2}^{0}$ and not being wellresided is $\Sigma_{3}^{0}$ (we use the negation). Since the complexity of these properties is at most $\Sigma_{3}^{0}$, the construction of the desired automorphism can be placed on a tree. (We will not discuss the details on this placement nor of the construction of an automorphism of $\mathcal{E}$ but direct the reader to Harrington and Soare [1996b] or Cholak [1995].)

But before we can continue we need some notation: We will need a way to classify the complexity of automorphisms of $\varepsilon$. Let $\Phi$ be an automorphism of $\mathcal{E}$. We say $\Phi$ is a $\Delta_{n}^{0}$-automorphism if there is a $\Delta_{n}^{0}$ function $f$ such that $\Phi\left(W_{e}\right)={ }^{*} W_{f(n)}$. Then $f$ is called a presentation of $\Phi$. Two sets $A$ and $B$ are $\Delta_{3}^{0}$-automorphic iff there is a $\Delta_{3}^{0}$ automorphism $\Phi$ such that $\Phi(A)=B$. This method of classification can also be used for isomorphisms between $\mathcal{L}^{*}(A)$ and $\mathcal{L}^{*}(B) . \mathcal{L}^{*}(A)$ and $\mathcal{L}^{*}(B)$ are $\Delta_{3}^{0}$-isomorphic (written $\left.\mathcal{L}^{*}(A) \simeq{ }_{\Delta_{3}^{0}} \mathcal{L}^{*}(B)\right)$ iff there is an isomorphism $\Phi^{*}$ between $\mathcal{L}^{*}(A)$ and $\mathcal{L}^{*}(B)$ and a $\Delta_{3}^{0}$ function $f$ such that $\Phi^{*}\left(W_{e}^{*}\right)=W_{f(e)}^{*}$.

If an automorphism $\Phi$ is constructed on a tree then $\Phi$ has a presentation computable in the true path (which is $\Delta_{3}^{0}$ ). Hence all automorphism constructed in this way are $\Delta_{3}^{0}$ automorphisms (or even effective automorphisms). All of the known results except Theorems 3.2 and 4.2 involving automorphisms use $\Delta_{3}^{0}$-automorphisms.

The structure $\mathcal{E}$ has $2^{\aleph_{0}}$ automorphisms. Hence, there must be automorphisms which are not $\Delta_{3}^{0}$. Even with Lachlan's result there was some slight hope that the relation of whether two sets are automorphic would be $\Delta_{3}^{0}$ or at least arithmetical. But Theorem 3.2 shows this is not the case. Therefore we know that there are useful non- $\Delta_{3}^{0}$-automorphisms. But the proof of Theorem 3.2 does not allow us to clearly lay our hands on any such automorphisms. The automorphisms constructed for this theorem are based on complex 
arrangements of $\Delta_{3}^{0}$-isomorphisms. (The automorphisms constructed in Lachlan's proof are complex arrangements of effective isomorphisms.) Thus there is some hope that all automorphisms are just complex arrangements of $\Delta_{3}^{0}$-isomorphisms. The next collection of results provides us with some factual basis for this hope.

Let $\delta(A)=\{B: \exists C(B \sqcup C=A)\} . \&(A)$ is the set of splits of $A$ and $\delta(A)$ forms a Boolean algebra. $\mathcal{R}(A)=\{R: R \subseteq A$ and $R$ is computable $\}$. $\mathcal{R}(A)$ is the set of computable subsets of $A$ and is an ideal of $\&(A) . \varsigma_{\mathcal{R}}(A)$ is the quotient structure $\&(A)$ modulo $\mathcal{R}(A)$. If $W \in S(A)$ then let $W^{R(A)}$ be the equivalence class of $W$ in $\delta_{\mathcal{R}}(A)$. $\S_{\mathcal{R}}(A)$ is a Boolean algebra and is definable in $\mathcal{E}$ with a parameter for $A$. If $A$ and $B$ are automorphic then the structures $\wp_{\mathcal{R}}(A)$ and $\wp_{\mathcal{R}}(B)$ are isomorphic structures. But something much stronger is true.

THEOREM 4.3 (Cholak and Harrington [n.d.a]). If $A$ and $B$ are automorphic then the structures $\varsigma_{\mathcal{R}}(A)$ and $\varsigma_{\mathcal{R}}(B)$ are $\Delta_{3}^{0}$-isomorphic structures (that is there is an isomorphism $\Psi$ between $\S_{\mathcal{R}}(A)$ and $\varsigma_{\mathcal{R}}(B)$ and $a \Delta_{3}^{0}$-function $f$ such that for $W_{e} \in \&(A), W_{f(e)}$ is in $\Psi\left(W^{\mathcal{R}(A)}\right)$; and we will write this as $\left.8_{\mathcal{R}}(A) \simeq_{\Delta_{3}^{0}} \delta_{\mathcal{R}}(B)\right)$.

By Theorem 3.2 we cannot go from a $\Delta_{3}^{0}$ isomorphism from $\varsigma_{\mathcal{R}}(A)$ to $\varsigma_{\mathcal{R}}(B)$ to an automorphism of $\mathcal{E}$ taking $A$ to $B$. But there is the hope that we can add extra conditions to this $\Delta_{3}^{0}$ isomorphism to help construct the desired automorphism. Theorem 4.3 also provides evidence that we are limited to our current methods in how we can construct automorphisms of $\mathcal{E}$. This is certainly the case for hhsimple sets:

THEOREM 4.4. Let $H_{1}$ and $H_{2}$ be hhsimple. $H_{1}$ and $H_{2}$ are automorphic iff they are $\Delta_{3}^{0}$-automorphic iff $\mathcal{L}^{*}\left(H_{1}\right) \simeq{ }_{\Delta_{3}^{0}} \mathcal{L}^{*}\left(H_{2}\right)$.

Theorem 4.3 will also have an impact in showing that certain sets cannot be automorphic. Most such results go more or less as follows: First show that the sets $A$ and $B$ cannot be $\Delta_{3}^{0}$-automorphic. Then use the failure to be $\Delta_{3}^{0}$-automorphic to find a definable property $P$ true of $A$ but not of $B$. By using Theorem 4.3, perhaps we can formalize this process. If not then at least we can use Theorem 4.3 to diagonalize against all $\Delta_{3}^{0}$-isomorphisms and show that certain sets are not automorphic without producing a definable difference.

An announcement of Theorems 4.3 and 4.4 and some other interesting results and examples can be found in Cholak and Harrington [n.d.b].

Perhaps our current understanding of automorphisms of $\mathscr{E}$ may be helpful in other areas, such as the computably enumerable degrees. In our constructions we have a notion of an $e$-state; enough information, if correctly acted on, to build a partial automorphism between the first $e$ sets. We know that if we act properly, i.e. make moves to copy the well-visited and well-resided states, we can extend our actions to handle all $(e+1)$-states.

As we mentioned earlier, the normal back and forth argument fails. Back and forth arguments fail because we do not know anything about the $n$-types of $\mathcal{E}$.

Question 4.5. Describe the n-types of $\mathbb{E}$.

The next definition captures $\alpha$ moves of the standard back and forth argument (see Barwise [1973] or Ash and Knight [2000]).

Definition 4.6. $\quad$ (i) $\vec{A} \leq 1 \vec{B}$ iff every $\Sigma_{1}$ formula realized by $\vec{B}$ is realized by $\vec{A}$.

(ii) $\vec{A} \leq_{\beta} \vec{B}$ iff for all $\vec{D}, \delta$ if $1 \leq \delta<\beta$ there is a $\vec{C}$ such that $\vec{B}, \vec{D} \leq_{\delta} \vec{A}, \vec{C}$.

Question 4.7 (Knight). When is $\vec{A} \leq_{\beta} \vec{B}$ ? 
If for all computable $\alpha, A \equiv_{\alpha} B\left(A \leq_{\alpha} B\right.$ and $\left.B \leq_{\alpha} A\right)$ then $A$ and $B$ are automorphic (see Ash and Knight [2000]). So, by Theorem 3.2, for all computable $\alpha$, there are automorphic $A$ and $B$ such that $A \not_{\alpha} B$.

\section{Upward and downward cones}

We want to turn to computational complexity issues. First, we will consider the property of being automorphic to a complete set. This issue first arose because of Post's Program. One version of Post's Program is to find a definable property $Q$ in $\&$ such that if $Q(A)$ then $Q$ is incomplete. By Harrington and Soare [1991] this has a positive solution: There is a property $Q(A)$ such that there is an $A$ where $Q(A)$ holds and if $Q(A)$ holds then $A$ is not automorphic to a complete set.

THEOREM 5.1. All sets listed in the first paragraph of Section 3 (the references are the same as the references listed there) and the almost prompt sets (Harrington and Soare [1996b]) are automorphic to a complete set B.

This gives rises to the question of which sets are automorphic to complete sets.

QUESTION 5.2. (i) Characterize all sets automorphic to complete sets.

(ii) Is $\left\{e: W_{e}\right.$ is automorphic to a complete set $\} \Sigma_{1}^{1}$-complete?

In terms of the automorphism results, sending $A$ to a complete set $B$ means that we can add numbers to $B$ at any time and continue to build the automorphism. Harrington showed [see Harrington and Soare, 1996b] that for all incomplete $\mathbf{d}$ and all noncomputable $A$, there is a $B$ such that $A$ and $B$ are automorphic and $B \not_{T}$ d. But this construction also involves adding things to $B$. Cholak [1995] and, independently, Harrington and Soare [1996b] showed that every noncomputable set is automorphic a high set $B$. Hence we can pump up the degree in terms of jump class. Again this construction involved adding numbers to $B$. Given a random $A$, we have very little control over the actual degree of $B$. Controlling the degree of $B$ would involve restraining numbers from $B$. The question remains: Can we avoid downward cones?

Question 5.3. Let $A$ be incomplete. Does there exists a $B$ such that $A \simeq B$ and $A \not \nless_{T} B ?$

One might ask what is the complexity of the index set of those $A$ s that are automorphic to a $B$ which cannot Turing compute $A$ ? In fact, we can always turn most of our questions into index set questions.

\section{Invariant degree classes}

Martin [1966] showed that the degrees of the maximal sets are exactly the high degrees. Lachlan [1968a] and Shoenfield [1976] showed that the degrees of sets without maximal supersets are exactly the nonlow 2 degrees. So the high degrees and the nonlow 2 degrees are invariant. In addition, $\mathbf{L}_{\mathbf{0}}=\mathbf{0}$ and $\overline{\mathbf{L}_{\mathbf{0}}}=\mathbf{R}-\mathbf{0}$ are invariant as witnessed by the computable sets and noncomputable sets, respectively.

Harrington in unpublished work showed that the property of being creative is elementarily definable in $\mathcal{E}$ [see Soare, 1987, XV.1.1]. Hence $\mathbf{H}_{\mathbf{0}}$ is invariant. Harrington and Soare [1996b] showed that every prompt set is automorphic to a complete set and hence $\overline{\mathbf{H}_{0}}$ is noninvariant. Maass, Shore and Stob [1981] showed that there is a definable class of sets (the promptly simple sets - see below) which splits all jump classes. Also, in as yet unpublished work, Harrington and Soare [see Harrington and Soare, 1996a, Corollary 4.4] 
have shown that $\overline{\mathbf{L}_{\mathbf{1}}}$ is not invariant. They prove this by showing there is a properly low 2 degree $\mathbf{d}$ such that if $A \leq_{T} \mathbf{d}$ then there is a low $B$ such that $A$ and $B$ are automorphic. (Note the similarities between this and Question 6.5.) The theorem that every noncomputable set is automorphic to a high set implies that no downward closed jump class is invariant.

It is known that the prompt degrees are invariant: Maass [1983] showed that all promptly simple sets with semilow complements are automorphic. Maass et al. [1981] show that every promptly simple set has the splitting property (a definable property, see Maass et al. [1981] for details) and every set which has the splitting property is prompt. Now it is enough to show that there is a promptly simple set with semilow complement in every prompt degree. One such proof can be found in Wald [1999, Theorem 1.4.1]. We cannot help but point out that the prompt degrees have a dynamic definition and are naturally definable in the computably enumerable degrees [for more details see Soare, 1987, $\mathrm{XIII}$.

The most recent result on invariant jump classes is:

THEOREM 6.1 (Cholak and Harrington [n.d.c]). For all $n \geq 2$, the high $\left(\overline{l o w_{n}}\right)$ com- $^{-}$ putably enumerable degrees are invariant.

We would like to look slightly closer at this result and some of its corollaries. (See the paper Cholak and Harrington [n.d.c] or the announcement Cholak and Harrington [n.d.b] for more details.)

Theorem 6.2 (Cholak and Harrington [n.d.c]). Let $\mathrm{C}=\{\mathbf{a}: \mathbf{a}$ is the Turing degree of a $\Sigma_{3}^{0}$ set $\left.J \geq_{T} \mathbf{0}^{\prime \prime}\right\}$. Let $\mathcal{D} \subseteq \mathcal{C}$ be such that $\mathscr{D}$ is upward closed. Then there is an $\mathcal{L}(A)$ property $\varphi_{\mathbb{D}}(A)$ such that $D^{\prime \prime} \in \mathbb{D}$ iff there is an $A$ such that $A \equiv_{T} D$ and $\varphi_{\mathscr{D}}(A)$.

COROLlary 6.3. Let $\mathcal{F}$ be a class of computably enumerable degrees such that if $\mathbf{a} \in \mathcal{F}$ and $\mathbf{a}^{\prime \prime} \leq_{T} \mathbf{b}^{\prime \prime}$ then $\mathbf{b} \in \mathcal{F}$. Then $\mathcal{F}$ is invariant.

Corollary 6.4. If $\mathbf{a}^{\prime \prime}>\mathbf{b}^{\prime \prime}$ then there is an $A \in \mathbf{a}$ such that for all $B \in \mathbf{b}, A \not B$ (in fact, $\mathcal{L}(A)$ and $\mathscr{L}(B)$ are not isomorphic).

More or less we have shown that the double jump can be encoded into $\&$. What about the single jump? The following question is the strongest possible negation of encoding the single jump in the above fashion (see Corollary 6.4) and is a generalization of the result of Harrington and Soare that $\overline{\mathbf{L}_{\mathbf{1}}}$ is noninvariant.

QuesTION 6.5. Let $J$ be computably enumerable in and above $\mathbf{0}^{\prime \prime}$. There are degrees $\mathbf{a}$ and $\mathbf{b}$ such that $\mathbf{a}^{\prime} \neq \mathbf{b}^{\prime}, \mathbf{a}^{\prime \prime} \equiv_{T} \mathbf{b}^{\prime \prime} \equiv_{T} J$, and for all $A \leq_{T}$ a there is a $B$ such that $B \leq_{T} \mathbf{b}$ and $A \simeq B$.

The "no fat orbit" result of Downey and Harrington [1996] (this result will be discussed later) says that the degrees in the above conjecture most likely are both prompt or both tardy (not prompt). So the fact that the prompt degrees are invariant provides some evidence that the conjecture should be true. In addition, we have some partial results in this direction: For example, in Cholak [1995], it is shown, for all $A$ and for all $\mathbf{H}_{\mathbf{1}}$ degrees $\mathbf{h}$ there is a $B \in \mathbf{h}$ such that $\mathcal{L}^{*}(A) \simeq \mathcal{L}^{*}(B)$. Recently Harrington has announced a generalization of the above theorem:

Theorem 6.6 (Harrington). For all $A$ and degrees $\mathbf{d}$ if $A^{\prime} \leq_{T} \mathbf{d}^{\prime}$ there is a $B \in \mathbf{d}$ such that $\mathcal{L}^{*}(A) \simeq \mathscr{L}^{*}(B)$. 
One might also want to try to combine a positive answer to Question 6.5 with Shore's noninversion theorem for the jump [Shore, 1988] or the result of Cooper [1989] that there is a degree computably enumerable in and above $\mathbf{0}^{\prime}$ which is not the jump of a tardy degree. In addition, one could add other degree theoretic conditions on $\mathbf{a}$ and $\mathbf{b}$.

By Corollary 6.3, $\mathbf{H}_{\omega}=\cup_{n \in \omega} \mathbf{H}_{\mathbf{n}}$ is invariant. Solovay has shown $\mathbf{H}_{\omega}$ is $\Sigma_{\omega+1}^{0}$-complete and hence nonarithmetical [see Soare, 1987, XII.4.14]. Let $\mathcal{C}$ be the class of computably enumerable sets witnessing that $\mathbf{H}_{\omega}$ is invariant. So a computably enumerable degree $\mathbf{d}$ is in $\mathbf{H}_{\omega}$ if there is a computably enumerable set $A$ in both $\mathbf{d}$ and $\mathcal{C}$. Whether $A$ is in $\mathbf{d}$ is arithmetical. So whether $A$ is in $\mathcal{C}$ cannot be arithmetical. Hence $\mathcal{C}$ is not definable in $\mathcal{E}$ by a $\mathcal{L}_{\omega, \omega}$ formula. (Otherwise it would be arithmetical.)

The above class $\mathcal{C}$ provides another ${ }^{1}$ negative answer to Shoenfield's question if every class $\mathcal{C}$ of computably enumerable sets closed under automorphic images is definable in $\mathscr{E}$ by a $\mathcal{L}_{\omega, \omega}$ formula. We note that such a $\mathcal{C}$ is definable in $\mathcal{E}$ by a $\mathcal{L}_{\omega_{1}, \omega}$ formula: $\mathcal{C}$ is a countable union of orbits. Each orbit is definable by a $\mathcal{L}_{\omega_{1}, \omega}$ formula. So $\mathcal{C}$ is definable by the countable disjunction the formulas defining the orbits in $\mathcal{C}$.

The formula $\varphi_{D}(A)$ in Theorem 6.2 is a $\mathcal{L}_{\omega_{1}, \omega}$ formula. One might ask if this formula can be improved to an elementary formula. The above work concerning $\mathbf{H}_{\omega}$ shows that in general this is not possible. But it leaves open the possibility that the complexity of the defining formula can be decreased in some cases. Call a class $\mathscr{D}$ of computably enumerable degrees an elementarily definable invariant degree class if $\mathscr{D}$ is invariant and the class $\mathcal{C}$ of computably enumerable sets witnessing $\mathcal{D}$ is invariant is elementarily definable. The high degrees and the nonlow 2 degrees are both elementarily definable invariant degree classes. Are the high 2 degrees an elementarily definable invariant degree class?

Corollary 6.3 shows that any class of degrees $\mathcal{F}$ such that if $\mathbf{a} \in \mathcal{F}$ and $\mathbf{a}^{\prime \prime}=\mathbf{b}^{\prime \prime}$ then $\mathbf{b} \in \mathcal{F}$ is invariant. This is not true for the single jump, for example as the nonlow degrees are not invariant. But this says nothing about classes which are unrelated to the jump operator.

QUESTION 6.7. Characterize the (elementarily definable) invariant degree classes.

In particular:

QUESTION 6.8. Are the array noncomputable degrees invariant?

As evidence that the above question might have a positive answer, we note that in the collection of all $\Pi_{1}^{0}$ classes, Cholak, Coles, Downey and Herrmann [n.d.] have recently shown that the anc degrees are invariant.

But how should we approach this question? Downey, Jockusch and Stob [1990] showed that every nonlow 2 degree is anc but there are low $_{2}$ anc degrees. The nonlow 2 degrees are invariant. There are two proofs of this: First Lachlan [1968a] and Shoenfield [1976] showed that the degrees of atomless sets are exactly the nonlow 2 degrees and second, via Theorem 6.1. One could try to find another definable property which exactly describes the anc degrees. Or one could try to push the second proof through to work for the anc degrees. Both of these plans seem unlikely. The most likely outcome is there is an anc degree $\mathbf{d}$ such that every $A \in \mathbf{d}$ is automorphic to a nonanc set. This is similar to Question 6.5 and Harrington and Soare's result that there is properly low 2 degree $\mathbf{d}$ such that every $A \in \mathbf{d}$ is automorphic to a low set.

Downey and Harrington [1996] have shown that there is no fat orbit. That is, they showed there is a definable property $S(A)$, a prompt $\mathbf{L}_{\mathbf{1}}$ degree $\mathbf{d}_{\mathbf{1}}$, a prompt $\mathbf{H}_{\mathbf{2}}$ degree

\footnotetext{
${ }^{1}$ The work in Lempp [1987] and Nies [1997] also provides negative answers.
} 
$\mathbf{d}_{\mathbf{2}}$ greater than $\mathbf{d}_{\mathbf{1}}$, and a tardy $\mathbf{H}_{\mathbf{2}}$ degree e such that for all $E \leq_{T} \mathbf{e}, \neg S(E)$ and if $\mathbf{d}_{\mathbf{1}} \leq_{T} D \leq_{T} \mathbf{d}_{\mathbf{2}}$ then $S(D)$. This result points out the dichotomy between the prompt and tardy degrees. A corollary is that, except for the high degrees, no single orbit can witness that a member of the $\mathbf{H}_{n}, \mathbf{L}_{n}$ hierarchy is invariant. Downey and Harrington ask whether their result can be extended to include the case when $\mathbf{e}$ is high. Harrington and Cholak conjecture that this is not the case:

CONJECTURE 6.9 (Cholak-Harrington). For every noncomputable degree a there is a set A whose orbit contains every high degree.

All of the above theorems and questions explore invariant degree classes and orbits containing only degrees in these classes. The "no fat orbit" theorem says that we can separate certain degrees via orbits: we can separate some prompt degree from a tardy degree. A positive answer to the above conjecture implies that we cannot separate a random degree from any high degree via orbits. The following question generalizes this idea.

Question 6.10. Is there a tardy $\left(\mathbf{L}_{\mathbf{n}}, \overline{\mathbf{H}_{\mathbf{n}}}\right)$ set A whose orbit contains a set of every prompt $\left(\overline{\mathbf{L}_{\mathbf{n}}}, \mathbf{H}_{\mathbf{n}}\right)$ degree?

\section{Decidability and coding issues}

Harrington and Herrmann developed various coding methods in $\mathcal{E}^{*}$. They both were able to use their methods to show that the theory of $\mathcal{E}^{*}$ is undecidable [Herrmann, 1984; Harrington, 1983]. Harrington was able to extend his methods to show that the theory of $\mathcal{E}^{*}$ has degree $\mathbf{0}^{(\omega)}$ [see Cholak, 1994a; Harrington and Nies, 1998]. The coding method used for this result has been further developed in Harrington and Nies [1998], where is it is shown that the structure $(\omega,+, \times, 0,1, \leq)$ can be coded in $\mathcal{E}^{*}$ with parameters but one cannot code a linear order into $\mathcal{E}^{*}$ without parameters. Nies [1998] later sharpened this result to show that the standard model of arithmetic can be coded in $\mathcal{E}^{*}$ with 5 alternations of quantifiers and the $\Pi_{6}$-theory of $\mathcal{E}^{*}$ is undecidable.

QUESTION 7.1. How many alternations of quantifiers does it take to define a model of arithmetic? 4 or less?

On the decidable side, Lachlan [1968b] showed that the $\exists \forall$-theory of $\mathcal{E}^{*}$ is decidable (he included $0,1, \cup$ and $\cap$ in the language). This was later slightly expanded by Lerman and Soare [1980]. See Soare [1987, XVI.2] for an overview of these results.

QUeSTION 7.2. Is the $\forall \exists \forall$-theory of $\mathcal{E}^{*}$ decidable? At what exact level does the theory of $\mathcal{E}^{*}$ fail to be decidable?

We should mention that the proofs of Theorems 4.3 and 6.2 involve a new definable encoding. The actual details of this coding are complex. These details and some theorems concerning this coding will appear in Cholak and Harrington [n.d.c]. We feel that this coding has great potential. This coding is simpler to decode than the previous coding. More or less, it can be decoded at the $\Sigma_{3}^{A}$ level. Perhaps it can be used to answer the above two questions.

\section{Connections with the computably enumerable degrees?}

Even given Cooper's claim that there is an automorphism of the computably enumerable degrees, the biinterpretability conjecture with parameters remains a strong possibility to globally understand the computably enumerable degrees $(\mathcal{R})$ [see Cooper, 1999; Shore, 1999; Slaman, 1999]. 
One of the corollaries of the biinterpretability conjecture with parameters is that the underlying structure has at most countably many automorphisms. We know that $\&$ has $2^{\aleph_{0}}$ automorphisms; hence the biinterpretability conjecture with parameters for $\mathscr{E}$ fails. It would be nice to suggest a global paradigm for $\mathcal{E}$.

It is still open if $\mathcal{R}$ has $2^{\aleph_{0}}$ automorphisms. If so, we suggest that any paradigm to describe the computably enumerable degrees can be used to describe the computably enumerable sets and vice-versa.

We will close by pointing out one of the many similarities between the computably enumerable sets and degrees. By Corollary 6.3, any class of computably enumerable degrees closed under the double jump is invariant in $\&$. Nies, Shore and Slaman [1998] have shown that any relation on the computably enumerable degrees which is invariant (used in a different sense) under the double jump is definable in $\mathcal{R}$ iff it is definable in first order arithmetic. See Shore [2000] for more details.

\section{References}

Ash, C. J. and Knight, J. F. [2000]. Computable structures and the hyperarithmetical hierarchy. In prepartion. Barwise, J. [1973]. Back and forth through infinitary logic, pp. 5-34. MAA Studies in Math., Vol. 8.

Cholak, P. [1994a]. Notes on 3 theorems by Leo Harrington. Handwritten Notes.

Cholak, P. [1994b]. The translation theorem, Arch. Math. Logic 33: 87-108.

Cholak, P. [1995]. Automorphisms of the lattice of recursively enumerable sets, Mem. Amer. Math. Soc. 113(541): viii+151.

Cholak, P., Coles, R., Downey, R. and Herrmann, E. [n.d.]. Automorphisms of the lattice of $\Pi_{1}^{0}$ classes; perfect thin classes and anc degrees. Submitted, Draft available.

Cholak, P. and Downey, R. [n.d.]. Some orbits for $\mathcal{E}^{*}$. Preprint Available.

Cholak, P., Downey, R. and Harrington, L. A. [n.d.]. Automorphisms of the computably enumerable sets: $\Sigma_{1}^{1}$ completeness. In preparation.

Cholak, P. and Harrington, L. A. [n.d.a]. $\Delta_{3}^{0}$-automorphisms of the computably enumerable sets. In preparation.

Cholak, P. and Harrington, L. A. [n.d.b]. Definable encodings in the computably enumerable sets. Submitted, Draft available.

Cholak, P. and Harrington, L. A. [n.d.c]. On the definability of the double jump in the computably enumerable sets. Submitted, Draft available.

Cooper, S. [1999]. Local degree theory, in E. R. Griffor (ed.), Handbook of computability theory, Vol. 140 of Studies in Logic, North-Holland Publishing Co., chapter 4, pp. 121-154.

Cooper, S. B. [1989]. A jump class of noncappable degrees, J. Symbolic Logic 54: 324-353.

Downey, R. G., Jockusch, Jr., C. G. and Stob, M. [1990]. Array nonrecursive sets and multiple permitting arguments, in K. Ambos-Spies, G. H. Muller and G. E. Sacks (eds), Recursion Theory Week, Oberwolfach 1989, Vol. 1432 of Lecture Notes in Mathematics, Springer-Verlag, Heidelberg, pp. 141-174.

Downey, R. G. and Stob, M. [1990]. Automorphisms and splittings of recursively enumerable sets, Proceedings of the Fourth Asian Logic Conference, CSSK Centre, pp. 75-87.

Downey, R. and Harrington, L. [1996]. There is no fat orbit, Ann. Pure Appl. Logic 80(3): 277-289.

Friedberg, R. M. [1958]. Three theorems on recursive enumeration. I. decomposition. II. maximal set. III. enumeration without duplication., J. Symbolic Logic 23: 309-316.

Harrington, L. A. [1983]. The undecidability of the lattice of recursively enumerable sets. Handwritten Notes.

Harrington, L. A. and Nies, A. [1998]. Coding in the partial order of enumerable sets, Adv. Math. 133(1): 133162.

Harrington, L. A. and Soare, R. I. [1991]. Post's program and incomplete recursively enumerable sets, Proc. Nat. Acad. Sci. U.S.A. 88: 10242-10246.

Harrington, L. A. and Soare, R. I. [1996a]. Definability, automorphisms, and dynamic properties of computably enumerable sets, Bull. Symbolic Logic 2(2): 199-213. 
Harrington, L. A. and Soare, R. I. [1996b]. The $\Delta_{3}^{0}$-automorphism method and noninvariant classes of degrees, J. Amer. Math. Soc. 9(3): 617-666.

Herrmann, E. [1984]. The undecidability of the elementary theory of the lattice of recursively enumerable sets, Frege conference, 1984 (Schwerin, 1984), Akademie-Verlag, Berlin, pp. 66-72.

Herrmann, E. [1989]. Automorphisms of the lattice of recursively enumerable sets and hyperhypersimple sets, Logic, methodology and philosophy of science, VIII (Moscow, 1987), North-Holland, Amsterdam, pp. 179190.

Lachlan, A. H. [1968a]. Degrees of recursively enumerable sets which have no maximal supersets., J. Symbolic Logic 33: 431-443.

Lachlan, A. H. [1968b]. The elementary theory of the lattice of recursively enumerable sets, Duke Math. J. 35: $123-146$.

Lempp, S. [1987]. Hyperarithmetical index sets in recursion theory, Trans. Amer. Math. Soc. 303: 559-583.

Lerman, M., Shore, R. A. and Soare, R. I. [1978]. $r$-maximal major subsets, Israel J. Math. 31(1): 1-18.

Lerman, M. and Soare, R. I. [1980]. A decidable fragment of the elementary theory of the lattice of recursively enumerable sets, Trans. Amer. Math. Soc. 257(1): 1-37.

Maass, W. [1983]. Characterization of recursively enumerable sets with supersets effectively isomorphic to all recursively enumerable sets, Trans. Amer. Math. Soc. 279: 311-336.

Maass, W., Shore, R. A. and Stob, M. [1981]. Splitting properties and jump classes, Israel J. Math. 39: 210-224.

Martin, D. A. [1966]. Classes of recursively enumerable sets and degrees of unsolvability, Z. Math. Logik Grundlag. Math. 12: 295-310.

Nies, A. [1997]. Intervals of the lattice of computably enumerable sets and effective boolean algebras, Bull. Lond. Math. Soc. 29: 683-92.

Nies, A. [1998]. Coding methods in computability theory and complexity theory. Habilitationsschrift, Universität Heidelberg.

Nies, A., Shore, R. A. and Slaman, T. A. [1998]. Interpretability and definability in the recursively enumerable degrees, Proc. London Math. Soc. (3) 77(2): 241-291.

Shoenfield, J. R. [1976]. Degrees of classes of recursively enumerable sets, J. Symbolic Logic 41: 695-696.

Shore, R. [1999]. The recursively enumerable degrees, in E. R. Griffor (ed.), Handbook of computability theory, Vol. 140 of Studies in Logic, North-Holland Publishing Co., chapter 6, pp. 169-198.

Shore, R. [2000]. Natural definability in degree structures, in P. Cholak, S. Lemmp, M. Lerman and R. Shore (eds), Computability Theory and Its Applications: Current Trends and Open Problems, American Mathematical Society.

Shore, R. A. [1988]. A noninversion theorem for the jump operator, Ann. Pure Appl. Logic 40: 277-303.

Slaman, T. [1999]. The global structure of the Turing degrees, in E. R. Griffor (ed.), Handbook of computability theory, Vol. 140 of Studies in Logic, North-Holland Publishing Co., chapter 5, pp. 155-168.

Soare, R. I. [1974]. Automorphisms of the lattice of recursively enumerable sets I: maximal sets, Ann. of Math. (2) 100: 80-120.

Soare, R. I. [1987]. Recursively Enumerable Sets and Degrees, Perspectives in Mathematical Logic, Omega Series, Springer-Verlag, Heidelberg.

Soare, R. I. [2000]. Extensions, automorphisms, and definability, in P. Cholak, S. Lemmp, M. Lerman and R. Shore (eds), Computability Theory and Its Applications: Current Trends and Open Problems, American Mathematical Society.

Wald, K. [1999]. Automorphism and noninvariant properites of the computably enumerable sets, $\mathrm{PhD}$ thesis, University of Chicago.

Department of Mathematics, University of Notre Dame, Notre Dame, IN 46556-5659

E-mail address: Peter. Cholak.1@nd.edu 